\title{
FUNCIONES DE ALTURA TOTAL Y AREA DE COPA PARA LENGA Nothofagus pumilio (Poepp. et Endl.) Krasser) EN LAGO GENERAL VINTTER - CERRO COLORADO, PROVINCIA DE CHUBUT - ARGENTINA. ALCANCES POR CLASES DE EXPOSICION Y ALTITUD C. Fernández y G. Martínez Pastur, CADIC - Programa de Recursos Vegetales. cc 92 (9410) Ushuaia, Prov. de Tierra del Fuego Argentina; F. Boyeras; P. Peri, Ingeniero Forestal, UNLP, Argentina.
}

\section{RESUMEN}

Para lenga se ajustaron funciones de Altura Total y Area de Copa. Los datos provienen del Lago General Vintter y utilizan sólo al DAP como variable predictora. Los ajustes se hicieron mediante técnicas de regresión lineal simple y múltiple y fueron analizados estadística y gráficamente, dando resultados satisfactorios. Un modelo fue seleccionado para cada combinación de exposición y altitud, entre los que presentaron mejor aptitud y estadísticos. Entre los que respondieron de manera más satisfactoria se encuentran

$\begin{array}{ll}H T= & \text { a*DAP0.5 } \\ A C= & \text { a*DAP } \\ H T= & \text { Altura Total }(\mathrm{m}) \\ A C= & \text { Area de Copa }\left(\mathrm{m}^{2}\right) \\ D A P= & \text { Diámetro a la Altura del Pecho }(\mathrm{cm}) .\end{array}$

Posteriormente se diferenciaron estadísticamente los modelos seleccionados para las diferentes combinaciones de exposición y altitud. Se determinaron las pautas para la planificación en la toma de datos para la construcción de ecuaciones de Attura Total y Area de Copa.

Palabras clave: Lenga (Nothofagus pumilio), Funciones Attura, Funciones Area de copa 


\section{INTRODUCCION}

El presente estudio integra una serie de trabajos realizados en la orilla sur del Lago General Vintter y al pie del Cerro Colorado, en la Provincia del Chubut, Argentina.

La lenga Nothofagus pumilio (Poepp. et Endl.) Krasser es una especie de gran interes desde el punto de vista ecológico. El amplio rango latitudinal y altitudinal y la rigurosidad de las condiciones climáticas en que se desarrolla le confieren características de excepción en lo que respecta a su mecanismo biológico (Carabelli, 1991).

El área en estudio se encuentra entre los $43^{\circ} 57^{\prime}$ y los $44^{\circ} 04^{\prime} \mathrm{L}$. S. y los $71^{\circ} 33^{\prime}$ hasta los $71^{\circ} 43^{\prime} \mathrm{L}$. W., cubriendo una superficie de 5.800 ha de bosques, que corresponden al Tipo Forestal Lenga, Subtipo Forestal Bosque Puro de Lenga (Uriarte y Grosse, 1991).

La lenga crece desde las márgenes del Lago General Vintter (935 msnm) y la estepa hasta los valles y las laderas del Cerro Colorado. En los mejores sitios alcanza los 30 metros de altura total y $130 \mathrm{~cm}$ de DAP, estos corresponden a lugares húmedos de exposición Sur y baja altitud. Se observa un gradiente altitudinal en lo que respecta a las alturas dominantes. A los 1.300 msnm se presentan las lengas en "1" de menor altura y a los $1.400 \mathrm{msnm}$ se presenta en forma achaparrada (Moore, 1983) debido a los factores ambientales adversos. Las laderas de exposición Norte presentan un ambiente más seco con un sotobosque totalmente diferente y un bosque más abierto y enfermo.

Para la determinación de las Alturas Totales y las Areas de Copa, mediante las ecuaciones correspondientes, se utiliza el diámetro (DAP), ya que es una variable correlacionada con los parámetros a calcular (Clutter et al, 1983). Las muestras que se incluyen en una misma regresión deben tomarse teniendo en cuenta las condiciones del sitio (Clutter et al, 1983). Para esto, en el muestreo se consideraron como posibles variables de la calidad de sitio la exposición y la altitud (Puente et al., 1985; Donoso, 1985). Se desconoce si se obtienen diferencias significativas, al realizar el muestreo para la construcción de 
ecuaciones, sin tener en cuenta la exposición y la altitud.

Los únicos antecedentes que se encontraron en la bibliografía para la zona considerada, son estudios dasométricos e instalación de parcelas permanentes para el manejo del bosque de lenga (Orfila, 1987; Mutarelli y Orfila, 1971). No existiendo ecuaciones de Altura Total y Area de Copa.

\section{OBJETIVOS}

- Elaborar un conjunto de ecuaciones de Altura Total para lenga, que permita estimar este parametro en ejemplares que se encuentren en distintas exposiciones y altitudes.

Elaborar un conjunto de ecuaciones de Area de Copa para Lenga que permita estimar este parametro en ejemplares que se encuentren en distintas exposiciones y altitudes.

Determinar si existen diferencias estadísticas significativas entre los modelos de las distintas combinaciones de exposición y altitud, de manera de descubrir los alcances de las mismas y acotar su rango de utilización.

Dar pautas para la planificación en la toma de datos para la elaboración de ecuaciones de Altura Total y Area de Copa para lenga en la zona bajo estudio.

\section{MATERIALES Y METODOS}

Estimación de la Altura Total y Area de Copa

La toma de datos se realizó obteniendo muestras homogéneamente distribuidas en la superficie bajo estudio. La selección se hizo tratando de cubrir la variación diamétrica para cada combinación de exposición y altitud. 
A los árboles muestreados se les midió diámetro en cruz con forcípula a 130 $\mathrm{cm}$ de altura, altura total con clinómetro y diámetro de copa en dos sentidos, con cinta métrica.

\section{Modelos Ajustados}

Se ensayaron funciones donde el DAP es la variable predictora. Los ajustes se realizaron en base a técnicas de regresión lineal y múltiple.

\section{Evaluación Estadistica}

Para evaluar y comparar el ajuste de los modelos ensayados y realizar su posterior selección, se consideraron los siguientes estimadores estadísticos:

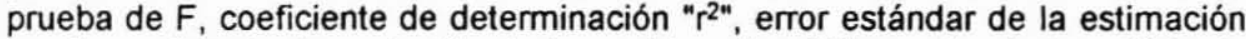
"ese", coeficiente de variación de los residuos "syx\%", prueba de $t$, análisis de los residuales "anare", ponderación "weight", Indice de Durbin-Watson, Indice de Spearman y el Indice de Furnival. De suma importancia se consideró también la simplicidad de los modelos (Little y Hills, 1985; Bonnier y Tedín, 1982; Chauchard, 1991; Yamane, 1979; Cantatore de Frank, 1983; Frield et al, 1991; ALder, 1980; Neter y Wassserman, 1973; Sokal y Rohlf, 1984; Clutter et al, 1983; Caillez, 1980).

\section{RESULTADOS Y DISCUSION}

\section{Muestra}

El total es de 332 individuos muestreados y los DAP mínimo y máximo obtenidos son, respectivamente, $10 \mathrm{~cm}$ y $130 \mathrm{~cm}$ (Cuadro 1). 


\section{Cuadro $\mathrm{N}^{\circ} 1$}

DISTRIBUCION DE LOS ARBOLES MUESTRA POR CLASE DIAMETRICA, ALTITUD Y EXPOSICION

\begin{tabular}{|l|c|ccccccc|c|}
\hline Exp & $\begin{array}{c}\text { Alt } \\
(\mathrm{msnm})\end{array}$ & \multicolumn{10}{|c|}{$\begin{array}{c}\text { DAP } \\
(\mathrm{cm})\end{array}$} & Total \\
\hline \multirow{3}{*}{ Este } & 1.000 & $<20$ & $20-30$ & $30-40$ & $40-50$ & $50-60$ & $60-70$ & $>70$ & \\
& 1.100 & 2 & 5 & 8 & 9 & 3 & 2 & 4 & 33 \\
& 1.200 & 4 & 2 & 5 & 6 & 9 & 2 & 2 & 28 \\
& 1.300 & 2 & 4 & 8 & 8 & 2 & 5 & 2 & 32 \\
\hline Sur & 1.000 & 2 & 4 & 6 & 5 & 5 & 3 & 7 & 32 \\
& 1.100 & 8 & 4 & 5 & 5 & 7 & 1 & 3 & 33 \\
& 1.200 & 0 & 3 & 10 & 4 & 8 & 1 & 0 & 26 \\
& 1.300 & 0 & 6 & 7 & 3 & 1 & 0 & 0 & 17 \\
\hline Norte & 1.000 & 5 & 3 & 5 & 6 & 8 & 2 & 2 & 31 \\
& 1.100 & 3 & 10 & 9 & 8 & 3 & 2 & 1 & 36 \\
& 1.200 & 4 & 12 & 11 & 2 & 1 & 1 & 0 & 31 \\
& 1.300 & 0 & 7 & 8 & 2 & 0 & 0 & 0 & 17 \\
\hline Total & & 32 & 62 & 91 & 60 & 47 & 19 & 21 & 332 \\
\hline
\end{tabular}

Modelos

Se ajustaron 14 modelos de ecuaciones para Altura Total y 13 modelos para estimación de Area de Copa. Entre los ensayados se encuentran los clásicos citados por la bibliografia y otros no tradicionales (Alder, 1980; Cailliez, 1980; Clutter et al, 1983; Little y Hills, 1985; Neter y Wasserman, 1973; Sokal y Rohlf, 1984; Gaillard de Benítez et al, 1988) (Cuadro 2) 


\section{Cuadro $\mathrm{N}^{\circ} 2$}

MODELOS DE ECUACIONES PARA ALTURA TOTAL Y AREA DE COPA

\begin{tabular}{|c|c|c|}
\hline $\mathrm{N}^{\circ}$ & Altura Total & Area de Copa \\
\hline 1 & $H T=a+b^{*} D$ & $A C=a+b \cdot D$ \\
\hline 2 & $H T=a+b^{*} D^{\wedge} 2$ & $A C=a+b^{*} D+c^{*} D^{\wedge} 2$ \\
\hline 3 & $H T=a+b^{*} D+c^{*} D^{\wedge} 2$ & $A C=a^{*} D$ \\
\hline 4 & $H T=a+b^{*} D^{\wedge} 2+c^{*} D^{\wedge} 3$ & $A C=a^{*} D+b^{*} D^{\wedge} 2$ \\
\hline 5 & $H T=a+b^{*} D+c^{*} D^{\wedge} 2+d^{*} D^{\wedge} 3$ & $A C=a^{*} D+b^{*} D^{\wedge} 0.5$ \\
\hline 6 & $H T=a^{*} D+b^{*} D^{\wedge} 2$ & $A C=a+b^{*} d^{\wedge} 2+c^{*} D^{\wedge} 3$ \\
\hline 7 & $H T=a^{*} D+b^{*} D^{\wedge} 2+c^{*} D^{\wedge} 3$ & $A C=a+b^{*} D^{\wedge} 2$ \\
\hline 8 & $H T=a^{*} D^{\wedge} 0.5$ & $A C=a+b^{*} D+c^{*} D^{\wedge} 2+d^{*} d^{\wedge} 3$ \\
\hline 9 & $H T=a^{*} D+b^{*} D^{\wedge} 0.5$ & $\operatorname{Ln} A C=a^{*} \operatorname{Ln}(D)+b^{\circ} D$ \\
\hline 10 & $H T=a^{*} D^{\wedge} 0.5+b^{*} 1 / D$ & $\operatorname{Ln} A C=a^{*} \operatorname{Ln}(D)+b^{*} D^{\wedge} 2$ \\
\hline 11 & $\operatorname{Ln} H T=a^{*} \operatorname{Ln}(D)$ & $\operatorname{Ln} A C=a^{*} \operatorname{Ln}(D)$ \\
\hline 12 & $\operatorname{Ln} H T=a+b^{*} \operatorname{Ln}(D)$ & $\operatorname{Ln} A C=a^{*} \operatorname{Ln}\left(D+D^{\wedge} 2\right)$ \\
\hline 13 & $\operatorname{Ln} H T=a^{*} \operatorname{Ln}(D)+b^{*} \operatorname{Ln}\left(D+D^{\wedge} 2\right)$ & $\operatorname{Ln} A C=a+b^{\circ} \operatorname{Ln}(D)$ \\
\hline 14 & $\operatorname{Ln} H T=a^{*} \operatorname{Ln}\left(D+D^{\wedge} 2\right)$ & $\operatorname{Ln} A C=a+b^{*} \operatorname{Ln}(D)$ \\
\hline
\end{tabular}

$H T=$ Altura Total $(m), A C=$ Area de Copa $\left(m^{2}\right): D=D A P(\mathrm{~cm})$

Una gran parte de los modelos, presentaron resultados satisfactorios. Aquellos que presentaron los mejores estadisticos y buena aptitud a través del ANARE, para cada combinación de exposición y altitud, son detallados en los Cuadros $\mathrm{N}^{\circ} \mathrm{s} 3$ a 8. 


\section{Cuadro $\mathrm{N}^{\circ} 3$}

VALORES DE LOS ESTADISTICOS OBTENIDOS PARA LOS MODELOS SELECCIONADOS PARA ALTURA TOTAL PARA LA EXPOSICION ESTE

\begin{tabular}{|c|c|c|c|c|c|c|c|c|c|c|c|c|}
\hline $\begin{array}{c}\text { Alt } \\
\text { (msnm) }\end{array}$ & $\begin{array}{l}\text { Mod } \\
\left(\mathrm{N}^{\circ}\right)\end{array}$ & R2 & $\begin{array}{l}\text { ESE } \\
(\mathrm{m})\end{array}$ & $\bar{F}$ & a & b & $\begin{array}{l}\# \\
\text { c }\end{array}$ & $\begin{array}{l}\text { Syx } \\
(\%)\end{array}$ & $\begin{array}{r}\text { Dur-V } \\
0\end{array}$ & & Furn & Pond \\
\hline \multirow[t]{4}{*}{1.000} & \multirow[t]{2}{*}{8} & 0,965 & 3,84 & 734,0 & \multicolumn{3}{|c|}{$\ddot{*}$} & 19,43 & 2,03 & $\bullet$ & 3,84 & - \\
\hline & & 0.955 & 0.11 & 683.6 & $*$ & & & 0.55 & 2.00 & • & 0.09 & $1 / D^{2}$ \\
\hline & \multirow[t]{2}{*}{13} & 0,995 & 1,62 & $3.218,0$ & $*$ & $*$ & & 7,11 & 1.90 & • & 1,77 & - \\
\hline & & 0,994 & 1,02 & $2.697,0$ & $\because$ & *. & & 0,34 & 1,84 & $\cdot$ & 0,88 & $1 / D^{2}$ \\
\hline \multirow[t]{4}{*}{1.100} & \multirow[t]{2}{*}{8} & 0,963 & 3,64 & 711,2 & $*$ & & & 19,97 & 1,49 & - & 3,64 & - \\
\hline & & 0,967 & 0,08 & 791.9 & $*$ & & & 0,44 & 1,75 & • & 0,06 & $1 / D^{2}$ \\
\hline & \multirow[t]{2}{*}{13} & 0,995 & 1,58 & $2.904,0$ & $*$ & *. & & 6,96 & 1,63 & • & 1,74 & - \\
\hline & & 0,995 & 1,02 & $3.050,0$ & 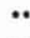 & *. & & 0,35 & 1,93 & • & 0,86 & $1 / D^{2}$ \\
\hline \multirow[t]{4}{*}{1.200} & \multirow[t]{2}{*}{6} & 0,963 & 3.18 & 414,4 & 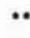 & .. & & 19,96 & 1.52 & • & 3,18 & - \\
\hline & & 0,962 & 0.09 & 398,0 & * & .. & & 0,55 & 1.99 & - & 0,07 & $1 / D^{2}$ \\
\hline & \multirow[t]{2}{*}{8} & 0,963 & 3.18 & 822,9 & 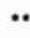 & & & 19,98 & 1.62 & - & 3,18 & . \\
\hline & & 0,952 & 0.09 & 615,7 & * & & & 0,62 & 1.62 & - & 0,07 & $1 / D^{2}$ \\
\hline \multirow[t]{4}{*}{1.300} & \multirow[t]{2}{*}{8} & 0,956 & 2,44 & 330,4 & $\because$ & & & 21,99 & 1,63 & $\cdot$ & 2.44 & - \\
\hline & & 0.956 & 0,44 & 323,8 & 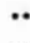 & & & 4,00 & 1,70 & $\cdot$ & 0,29 & $1 / D^{2}$ \\
\hline & \multirow[t]{2}{*}{11} & 0.990 & 1,74 & $2.620,0$ & $*$ & & & 10,09 & 1,36 & i & 2,02 & - \\
\hline & & 0.992 & 1.02 & $2.026,1$ & * & & & 0,42 & 1,63 & . & 0.77 & $1 / D^{2}$ \\
\hline
\end{tabular}

\# Para un valor de significancia del $95 \%$

-. altamente significativo, ${ }^{*}$ significativo, ns no significativo

(C) Para un punto de significancia del $5 \%$." valor superior al $D_{\mathrm{U}}$, i valor intermedio entre du y $D^{L}$, vi valor inferior al $d^{L}$ 


\section{Cuadro $\mathrm{N}^{\circ} 4$}

VALORES DE LOS ESTADISTICOS OBTENIDOS PARA LOS MODELOS SELECCIONADOS PARA ALTURA TOTAL PARA LA EXPOSICION SUR

\begin{tabular}{|c|c|c|c|c|c|c|c|c|c|c|c|c|}
\hline $\begin{array}{c}\text { Alt } \\
(\mathrm{msnm})\end{array}$ & $\begin{array}{l}\text { Mod } \\
\left(N^{0}\right)\end{array}$ & R2 & $\begin{array}{l}\text { ESE } \\
(\mathrm{m})\end{array}$ & $F$ & a & $\begin{array}{l}t \\
b\end{array}$ & $\#$ & $\begin{array}{l}\text { Syx } \\
(\%)\end{array}$ & $\begin{array}{c}\text { Dur- }-4 \\
\text { (2) } \\
\end{array}$ & & Furn & Pond \\
\hline \multirow{4}{*}{1.000} & 6 & 0,960 & 4,51 & 373,1 & $*$ & $*$ & & 20,95 & 1,87 & " & 4,51 & \\
\hline & & 0,938 & 0,13 & 235,5 & 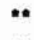 & ** & & 0,58 & 1,66 & • & 0,10 & $1 / D^{2}$ \\
\hline & 8 & 0,956 & 4,74 & 671,8 & ** & & & 22,03 & 1,69 & • & 4,74 & \\
\hline & & 0,936 & 0.13 & 455,3 & .. & & & 0,58 & 1,39 & $\mathrm{i}$ & 0,11 & $1 / D^{2}$ \\
\hline \multirow[t]{4}{*}{1.100} & 8 & 0,967 & 3,01 & 948,5 & .. & & & 19,28 & 2,69 & • & 3,01 & \\
\hline & & 0,967 & 0,09 & 941,5 & *. & & & 0,58 & 2,25 & • & 0,07 & $1 / D^{2}$ \\
\hline & 13 & 0,994 & 1,62 & $2.711,2$ & *. & •• & & 7,81 & 2,40 & • & 1,77 & - \\
\hline & & 0,992 & 1,02 & $1.871,8$ & $\ddot{*}$ & •• & & 0,37 & 1,95 & • & 0,88 & $1 / D^{2}$ \\
\hline \multirow[t]{4}{*}{1.200} & 10 & 0,982 & 2,21 & 713,4 & $\ddot{*}$ & • & & 13,53 & 1,89 & • & 2,21 & 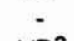 \\
\hline & & 0,985 & 0,05 & 814,2 & $\ddot{*}$ & $\ddot{*}$ & & 0,31 & 1,95 & • & 0,04 & $1 / D^{2}$ \\
\hline & 13 & 0,998 & 1,38 & $5.150,1$ & $\ddot{*}$ & $*$ & & 5,03 & 1,95 & • & 1,54 & 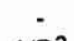 \\
\hline & & 0,998 & 1,02 & $5.928,6$ & $\ddot{*}$ & "* & & 0,36 & 1,97 & • & 0,85 & $1 / D^{2}$ \\
\hline \multirow{4}{*}{1.300} & 8 & 0,972 & 1,62 & 550,7 & ** & & & 17,50 & 2,06 & • & 1,62 & 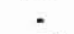 \\
\hline & & 0,972 & 0,05 & 545,5 & $*$ & & & 0,56 & 1,82 & • & 0,03 & $1 / D^{2}$ \\
\hline & 11 & 0,993 & 1,55 & $2.208,2$ & $*$ & & & 8,58 & 1,66 & • & 1,77 & 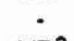 \\
\hline & & 0,992 & 1,02 & $2.010,1$ & * & & & 0,45 & 1,43 & $\cdot$ & 0,77 & $1 / D^{2}$ \\
\hline
\end{tabular}

\# Para un valor de significancia del 95\%

*. altamente significativo, " significativo, ns no significativo

(2) Para un punto de significancia del $5 \%$. valor superior al $\mathrm{D}_{\mathrm{u}}$, i valor intermedio entre du y $D^{L}$, vi valor inferior al $d^{L}$ 


\section{Cuadro $\mathrm{N}^{\circ} 5$}

VALORES DE LOS ESTADISTICOS OBTENIDOS PARA LOS MODELOS SELECCIONADOS PARA ALTURA TOTAL PARA LA EXPOSICION NORTE

\begin{tabular}{|c|c|c|c|c|c|c|c|c|c|c|c|c|}
\hline $\begin{array}{c}\text { Alt } \\
(\mathrm{msnm})\end{array}$ & $\begin{array}{l}\text { Mod } \\
\left(\mathrm{N}^{\circ}\right)\end{array}$ & R2 & $\begin{array}{l}\text { ESE } \\
\text { (m) }\end{array}$ & $F$ & a & b & $\begin{array}{l}\# \\
\text { c }\end{array}$ & $\begin{array}{l}\text { Syx } \\
(\%)\end{array}$ & $\begin{array}{r}\text { Dur-h } \\
\text { Q }\end{array}$ & & Furn & Pond \\
\hline \multirow[t]{4}{*}{1.000} & \multirow[t]{2}{*}{6} & 0,940 & 4,38 & 235,4 & $\cdots$ & $\cdots$ & & 26,51 & 1,52 & $\bullet$ & 4,38 & - \\
\hline & & 0,952 & 0,10 & 299,9 & ** & .• & & 0,62 & 1,65 & - & 0,08 & $1 / D^{2}$ \\
\hline & \multirow[t]{2}{*}{14} & 0,990 & 1,86 & $3.265,2$ & .. & & & 9,89 & 1,43 & $\mathbf{i}$ & 2,04 & - \\
\hline & & 0,992 & 1,17 & $3.959,0$ & *• & & & 2,56 & 1,87 & $\cdot$ & 1,01 & $1 / D^{2}$ \\
\hline \multirow[t]{4}{*}{1.100} & \multirow[t]{2}{*}{8} & 0,946 & 2,89 & 617,8 & *. & & & 24,66 & 1,33 & vi & 2,89 & - \\
\hline & & 0,933 & 0,10 & 487,4 & $\bullet$ & & & 0,84 & 1,23 & vi & 0.08 & $1 / D^{2}$ \\
\hline & \multirow[t]{2}{*}{13} & 0,988 & 1,86 & 1. 478,0 & $\cdot$ & • & & 11,22 & 1,28 & vi & 1,99 & - \\
\hline & & 0,988 & 1,02 & $1.545,0$ & $*$ & ** & & 0,42 & 1,36 & i & 0,89 & $1 / D^{2}$ \\
\hline \multirow[t]{4}{*}{1.200} & \multirow[t]{2}{*}{10} & 0.982 & 1,33 & 840,9 & *. & *. & & 13,61 & 2.09 & - & 1,33 & - \\
\hline & & 0,984 & 0,05 & 914,4 & *. & * & & 0,49 & 2,03 & $\cdot$ & 0,04 & $1 / D^{2}$ \\
\hline & \multirow[t]{2}{*}{13} & 0,996 & 1,38 & 3.827 .5 & * & $*$ & & 6,18 & 2,06 & $\cdot$ & 1,49 & - \\
\hline & & 0,996 & 1,02 & $4.193,6$ & .. & *. & & 0,44 & 1,99 & • & 0,88 & $1 / D^{2}$ \\
\hline \multirow[t]{4}{*}{1.300} & \multirow[t]{2}{*}{8} & 0,974 & 1.85 & 592,6 & .. & & & 16,99 & 1,76 & • & 1,85 & - \\
\hline & & 0,969 & 0,07 & 492,9 & .. & & & 0,60 & 1,85 & $\cdot$ & 0.05 & $1 / D^{2}$ \\
\hline & \multirow[t]{2}{*}{11} & 0,994 & 1,51 & 2.859 .0 & *. & & & 7,59 & 1,61 & - & 1,74 & - \\
\hline & & 0.993 & 1.02 & $2.361,3$ & *. & & & 0,42 & 1,67 & - & 0,78 & $1 / D^{2}$ \\
\hline
\end{tabular}

\# Para un valor de significancia del 95\%

* altamente significativo, " significativo, ns no significativo

(C) Para un punto de significancia del $5 \%$. valor superior al $D_{\mathrm{u}}$, i valor intermedio entre du y $D^{L}$, vi valor inferior al $d^{L}$ 


\section{Cuadro $\mathrm{N}^{\circ} 6$}

\section{VALORES DE LOS ESTADISTICOS OBTENIDOS PARA LOS MODELOS} SELECCIONADOS PARA AREA DE COPA PARA LA EXPOSICION ESTE

\begin{tabular}{|c|c|c|c|c|c|c|c|c|c|c|c|c|}
\hline $\begin{array}{c}\text { Alt } \\
\text { (msnm) }\end{array}$ & $\begin{array}{l}\text { Mod } \\
\left(N^{\circ}\right)\end{array}$ & $\mathrm{R} 2$ & $\begin{array}{l}\text { ESE } \\
(m)\end{array}$ & $F$ & a & $\begin{array}{l}t \\
b\end{array}$ & C & $\begin{array}{l}\text { Syx } \\
(\%)\end{array}$ & $\begin{array}{r}\text { Dur-V } \\
\text { Q }\end{array}$ & & Furn & Pond \\
\hline \multirow[t]{3}{*}{1.000} & 4 & 0,935 & 17,9 & 232,3 & $\ddot{*}$ & .. & & 33,12 & 1,81 & - & 17,9 & in \\
\hline & 11 & 0 & 0,4 & 1 & .. & 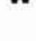 & & $\begin{array}{r}0,74 \\
13,45\end{array}$ & 1,71 & - & 0,3 & $D^{2}$ \\
\hline & 11 & 0,984 & 3,2 & $1.986,6$ & .. & & & 13,45 & 1,41 & i & 3,6 & 2 \\
\hline \multirow{4}{*}{1.100} & 3 & 0915 & 138 & 2911 & .• & & & 3284 & 178 & - & 138 & 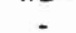 \\
\hline & & 0,901 & 0,3 & 250,5 & $\bullet$ & & & 0,73 & 2,08 & - & 0,2 & $1 / D^{2}$ \\
\hline & 11 & 0,994 & 2,0 & $4.905,6$ & - & & & 8,24 & 2,09 & $*$ & 2,3 & - \\
\hline & & 0,992 & 1,3 & $3.789,0$ & $\bullet$ & & & 2,75 & 2,23 & * & 1,1 & $1 / D^{2}$ \\
\hline \multirow[t]{4}{*}{1.200} & 11 & 0,984 & 2,5 & $1.985,6$ & $*$ & & & 11,71 & 2,18 & • & 2,8 & - \\
\hline & & 0,976 & 1,3 & $1.280,1$ & $*$ & & & 2,93 & 2,56 & i & 1,2 & $1 / D^{2}$ \\
\hline & 12 & 0.985 & 2,5 & $1.983,6$ & $\bullet$ & & & 11,71 & 2,17 & - & 2,8 & - \\
\hline & & 0.977 & 1,3 & $1.300,9$ & $*$ & & & 2,93 & 2.59 & $\mathrm{i}$ & 1,2 & $1 / D^{2}$ \\
\hline \multirow[t]{4}{*}{1.300} & 3 & 0,811 & 11,9 & 64,3 & $\bullet$ & & & 52,25 & 1.80 & - & 11,9 & - \\
\hline & & 0,828 & 0,3 & 72,4 & $\bullet$ & & & 1,47 & 1,69 & - & 0,2 & $1 / D^{2}$ \\
\hline & 11 & 0,962 & 4,0 & 389,8 & ** & & & 20,43 & 2,06 & - & 4,9 & - \\
\hline & & 0,970 & 1,3 & 499,0 & .. & & & 3,40 & 1,92 & 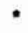 & 1.0 & $1 / D^{2}$ \\
\hline
\end{tabular}

\# Para un valor de significancia del $95 \%$

- altamente significativo, " significativo, ns no significativo

(C) Para un punto de significancia del $5 \%$. " valor superior al $D_{u}$, i valor intermedio entre du y $D^{L}$, vi valor inferior al $d^{L}$ 


\section{Cuadro $\mathrm{N}^{\circ} 7$} VALORES DE LOS ESTADISTICOS OBTENIDOS PARA LOS MODELOS
SELECCIONADOS PARA AREA DE COPA PARA LA EXPOSICION SUR

\begin{tabular}{|c|c|c|c|c|c|c|c|c|c|c|c|c|}
\hline $\begin{array}{c}\text { Alt } \\
(\mathrm{msnm})\end{array}$ & $\begin{array}{l}\text { Mod } \\
\left(N^{0}\right)\end{array}$ & $\overline{R 2}$ & $\begin{array}{l}\text { ESE } \\
(\mathrm{m})\end{array}$ & $\bar{F}$ & $a$ & $b$ & $\begin{array}{l}\# \\
c \\
\end{array}$ & $\begin{array}{l}\text { Syx } \\
(\%)\end{array}$ & $\begin{array}{r}\text { Dur-V } \\
\text { (Q }\end{array}$ & & Furn & Pond \\
\hline \multirow[t]{4}{*}{1.000} & 11 & 0,991 & 2,5 & $3.281,8$ & $*$ & & & 9,92 & 2,22 & $\cdot$ & 2,9 & - \\
\hline & & 0,991 & 1,3 & $3.467,6$ & 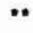 & & & 2,48 & 2,14 & - & 1,2 & $1 / D^{2}$ \\
\hline & 12 & 0,991 & 2,5 & $3.294,5$ & .* & & & 9,92 & 2,22 & - & 2,9 & - \\
\hline & & 0,991 & 1,3 & $3.514,4$ & *• & & & 2,48 & 2,16 & - & 1.2 & $1 / D^{2}$ \\
\hline \multirow[t]{4}{*}{1.100} & 11 & 0,947 & 4,0 & $1.139,3$ & $\bullet$ & & & 18,78 & 1,27 & ns & 4,5 & - \\
\hline & & 0,843 & 1,3 & 161,7 & $*$ & & & 3,13 & 1,56 & • & 1,1 & $1 / D^{2}$ \\
\hline & 12 & 0,974 & 4,0 & $1.141,2$ & • & & & 18,78 & 1,23 & ns & 4,5 & - \\
\hline & & 0,851 & 1,3 & 171,6 & $*$ & & & 3,13 & 1,52 & • & 1,1 & $1 / D^{2}$ \\
\hline \multirow[t]{4}{*}{1.200} & 11 & 0,981 & 3,2 & $1.280,3$ & ** & & & 14,34 & 2,25 & • & 3,7 & - \\
\hline & & 0,983 & 1.3 & $1.471,0$ & $*$ & & & 2,87 & 2,45 & - & 1,1 & $1 / D^{2}$ \\
\hline & 12 & 0,981 & 3,2 & $1.275,0$ & $*$ & & & 14,34 & 2,22 & • & 3,7 & - \\
\hline & & 0,983 & 1,3 & $1.465,4$ & $*$ & & & 2,87 & 2,40 & $\cdot$ & 1,1 & $1 / D^{2}$ \\
\hline \multirow[t]{4}{*}{1.300} & 3 & 0,949 & 4,7 & 294,8 & $*$ & & & 24,80 & 1,85 & $\cdot$ & 4,7 & - \\
\hline & & 0,943 & 0,1 & 264,9 & *. & & & 0,75 & 1,98 & * & 0,1 & $1 / D^{2}$ \\
\hline & 12 & 0,993 & 1,6 & $2.290,4$ & 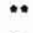 & & & 6,92 & 1,79 & • & 1,9 & - \\
\hline & & 0,993 & 1,3 & $2.292,9$ & .* & & & 3,46 & 1,91 & $\cdot$ & 1,0 & $1 / D^{2}$ \\
\hline
\end{tabular}

\# Para un valor de significancia del $95 \%$

* altamente significativo, " significativo, ns no significativo

(C) Para un punto de significancia del $5 \%$. " valor superior al $D_{u}$, i valor intermedio entre du y $D^{L}$, vi valor inferior al $d^{L}$ 


\section{Cuadro $\mathrm{N}^{\circ} 8$}

VALORES DE LOS ESTADISTICOS OBTENIDOS PARA LOS MODELOS SELECCIONADOS PARA ÁREA DE COPA PARA LA EXPOSICION NORTE

\begin{tabular}{|c|c|c|c|c|c|c|c|c|c|c|c|c|}
\hline $\begin{array}{c}\text { Alt } \\
(\mathrm{msnm})\end{array}$ & $\begin{array}{l}\text { Mod } \\
\left(N^{0}\right)\end{array}$ & R2 & $\begin{array}{l}\text { ESE } \\
(\mathrm{m})\end{array}$ & $F$ & a & b & C & $\begin{array}{l}\text { Syx } \\
(\%)\end{array}$ & $\begin{array}{r}\text { Dur-V } \\
\text { Q }\end{array}$ & & Furn & Pond \\
\hline \multirow[t]{4}{*}{1.000} & \multirow[t]{2}{*}{3} & 0,907 & 13,7 & 295,9 & \multicolumn{3}{|l|}{ 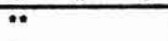 } & 35,45 & 2,07 & $\cdot$ & 13,7 & - \\
\hline & & 0,880 & 0,4 & 254,1 & *. & & & 0,92 & 2,26 & - & 0,3 & $1 / D^{2}$ \\
\hline & \multirow[t]{2}{*}{11} & 0,989 & 2,5 & $2.733,6$ & $* *$ & & & 11,53 & 2,11 & - & 2,8 & - \\
\hline & & 0,982 & 1,3 & $1.652,2$ & $*$ & & & 2,88 & 2,69 & $\mathrm{i}$ & 1,1 & $1 / D^{2}$ \\
\hline \multirow[t]{4}{*}{1.100} & \multirow[t]{2}{*}{3} & 0,885 & 9,5 & 270,7 & ** & & & 39,95 & 1,40 & i & 9,5 & - \\
\hline & & 0,850 & 0,3 & 199,2 & $\bullet$ & & & 1,26 & 1,28 & ns & 0,3 & $1 / D^{2}$ \\
\hline & \multirow[t]{2}{*}{12} & 0,979 & 3,2 & $1.596,0$ & - & & & 15,15 & 1,16 & ns & 3,5 & - \\
\hline & & 0,974 & 1,2 & $1.303,3$ & $\bullet$ & & & 0,50 & 1,17 & ns & 1,1 & $1 / D^{2}$ \\
\hline \multirow[t]{4}{*}{1.200} & \multirow[t]{2}{*}{3} & 0,883 & 7,2 & 226,1 & $\bullet$ & & & 40,85 & 1,76 & $\cdot$ & 7,2 & - \\
\hline & & 0,855 & 0,2 & 176,3 & $*$ & & & 0,01 & 1,94 & $\cdot$ & 0,2 & $1 / D^{2}$ \\
\hline & \multirow[t]{2}{*}{11} & 0,943 & 4,6 & 495,5 & $*$ & & & 25,39 & 1,62 & • & 5,0 & - \\
\hline & & 0,886 & 1,1 & 234,0 & $*$ & & & 1,23 & 1,50 & • & 1,0 & $1 / D^{2}$ \\
\hline \multirow[t]{4}{*}{1.300} & \multirow[t]{2}{*}{11} & 0,991 & 2,0 & $1.694,5$ & $* *$ & & & 10,49 & 1,80 & • & 2,4 & - \\
\hline & & 0,991 & 1,3 & $1.667,4$ & $*$ & & & 3.49 & 1.77 & $\cdot$ & 1,0 & $1 / D^{2}$ \\
\hline & \multirow[t]{2}{*}{12} & 0,991 & 2,0 & $1.688,7$ & $*$ & & & 10,49 & 1,80 & $\cdot$ & 2,4 & - \\
\hline & & 0,991 & 1,3 & $1.658,6$ & $*$ & & & 3,49 & 1,77 & $\cdot$ & 1,0 & $1 / D^{2}$ \\
\hline
\end{tabular}

\# Para un valor de significancia del $95 \%$

** altamente significativo," significativo, ns no significativo

(C) Para un punto de significancia del $5 \%$. valor superior al $D_{u}$, i valor intermedio entre du y $D^{L}$, vi valor inferior al $d^{L}$

Los valores de $\mathrm{F}$ para los modelos presentados, con y sin ponderación, se mostraron altamente significativos al $95 \%$ de significancia.

Los valores de significancia de $t$ fueron significativos $y$ altamente significativos.

El coeficiente de variación de los residuos presentó desde valores muy pequeños hasta valores del $40 \%$.

Algunos de los modelos ensayados presentaron autocorrelación de errores, ya que el Indice de Durbin-Watson obtenido en la regresión fue inferior al valor crítico teórico $\mathrm{d}^{\mathrm{L}}$. Otros se presentaron en la zona de indeterminación por lo que no se pudo comprobar la existencia de la autocorrelación de errores por este método. El resto no mostró evidencia de la autocorrelación de errores. 
El Indice de Sperman para cada variable independiente para los modelos finales seleccionados (Figuras Nos 9 a 14) dió diferencias no significativas en gran parte de los casos al aplicarse la prueba de $t$ con $n-2$ grados de libertad para un $95 \%$ de significancia. Por lo que fue rechazada la hipótesis que habia heterocedasticidad en los casos mencionados.

Al observar los $t$ obtenidos de los coeficientes de los modelos elegidos, se puede inferir que no hay multicolinealidad como lo sugiere la bibliografía (Cantatore de Frank, 1983).

El $r^{2}$ presentó buenos resultados entre los modelos finales seleccionados (Cuadros $\mathrm{N}^{\circ} \mathrm{s} 9$ a 14), pero algunos autores citan que el mismo puede aparacer sobreestimado por la ponderación (Alder, 1980; Chauchard, 1991). Por otra parte, regresiones en las cuales la misma variable dependiente ha sido sometida a diferentes transformaciones, no pueden compararse directamente mediante el $r^{2}$ (Alder, 1980). Por lo que se recurrió al Indice de Furnival, que tiene en cuenta las transformaciones que sufrieron la variable dependiente, el tamaño de la muestra, las ponderaciones aplicadas y el error estándar de la estimación.

\section{Modelos Finales Seleccionados}

Los modelos que presentaron los mejores estadisticos (para cada combinación de exposición y altitud), buena aptitud a través del ANARE y simplicidad, fueron seleccionados. Se los muestra en los Cuadros $N^{\circ} \mathrm{s} 9$ a 14.

Se tuvo en cuenta como principal estadistico al Indice de Furnival, seleccionando aquel modelo con menor valor. Las excepciones son los modelos de Area de Copa para exposición Norte a los 1100 msnm, que presentan problemas de autocorrelación de errores. 


\section{Cuadro $\mathrm{N}^{\circ} \mathrm{g}$}

MODELOS FINALES SELECCIONADOS PARA ALTURA TOTAL PARA LA EXPOSICION ESTE

\begin{tabular}{|lcc|}
\hline Altitudes (msnm) & & \\
\hline 1000 & $H T=3,05766^{*} D^{0,5}$ & Modelo 8 \\
& $\lim$ inf $=2,82129$ & $\lim$ sup $=3,29403$ \\
& $\operatorname{ta}=26,3558 \cdots$ & Weigth $=1 / D^{2}$ \\
\hline 1100 & $H T=2,793537^{*} D^{0,5}$ & Modelo 8 \\
& $\lim$ in $f=2,58981$ & $\lim$ sup $=2,99726$ \\
& $\operatorname{ta}=28,1419 \cdots$ & Weigth $=1 / D^{2}$ \\
\hline 1200 & $H T=2,480061^{*} D^{0,5}$ & Modelo 8 \\
& $\lim$ inf $=2,27617$ & lim sup $=2,68395$ \\
& $\operatorname{ta}=24,8134 \cdots$ & Weigth $=1 / D^{2}$ \\
\hline 1300 & $H T=2,007090^{*} D^{0,5}$ & Modelo 8 \\
& $\lim$ inf $=1,76819$ & lim sup $=2,24599$ \\
& $\operatorname{ta}=17,9115 \cdots$ & Weigth $=1 / D^{2}$ \\
\hline
\end{tabular}

$\mathrm{HT}=$ Altura Total $(\mathrm{m})$

$\mathrm{D}=$ Diámetro a la altura del pecho $(\mathrm{cm})$

$E S E=m$

$\lim$ sup y $\lim$ inf $=$ Intervalos de confianza al $95 \%$ de los coeficientes calculados.

* altamente significativos al $95 \%$ de significancia 


\section{Cuadro $\mathrm{N}^{\circ} 10$}

MODELOS FINALES SELECCIONADOS PARA ALTURA TOTAL PARA LA EXPOSICION SUR

\begin{tabular}{|c|c|c|}
\hline \multicolumn{3}{|c|}{ Altitudes (msnm) } \\
\hline \multirow[t]{5}{*}{1000} & $H T=0,737368^{*} D-0,005125^{\circ} D^{2}$ & Modelo 6 \\
\hline & $\lim$ inf $a=0,63146$ & $\lim$ sup $a=0,84327$ \\
\hline & $\lim \inf b=-0,00691$ & $\lim \sup b=-0,00334$ \\
\hline & $\mathrm{ta}=14,2229 *$ & \\
\hline & Weigth $=1 / D^{2}$ & \\
\hline \multirow[t]{3}{*}{1100} & $H T=2,601321 \cdot D^{0.5}$ & Modelo 8 \\
\hline & $\lim$ inf $=2.42859$ & $\lim$ sup $=2,77405$ \\
\hline & $\mathrm{ta}=30,6837 \cdots$ & Weigth $=1 / D^{2}$ \\
\hline \multirow[t]{5}{*}{1200} & $H T=1,955760 \cdot D^{0.5}+140,053948 / D$ & Modelo 10 \\
\hline & $\lim$ inf $a=1,549339$ & $\lim$ sup $a=2,36213$ \\
\hline & $\lim \inf b=48,40650$ & $\lim \sup b=231,7010$ \\
\hline & $\mathrm{ta}=9,9355 \cdots$ & $\mathrm{tb}=3,1548 \cdots$ \\
\hline & Weigth $=1 / D^{2}$ & \\
\hline \multirow[t]{3}{*}{1300} & $H T=1,658085 \cdot D^{0.5}$ & Modelo 8 \\
\hline & $\lim \inf =1,50756$ & $\lim$ sup $=1,80861$ \\
\hline & $\mathrm{ta}=23,3568 \cdots$ & Weigth $=1 / D^{2}$ \\
\hline
\end{tabular}

$H T=$ Altura Total $(m)$

$D=$ Diámetro a la altura del pecho $(\mathrm{cm})$

$E S E=m$

lim sup y lim inf $=$ Intervalos de confianza al $95 \%$ de los coeficientes calculados.

* altamente significativos al $95 \%$ de significancia 


\section{Cuadro $\mathrm{N}^{\circ} 11$}

\section{MODELOS FINALES SELECCIONADOS PARA ALTURA TOTAL PARA LA EXPOSICION NORTE}

\begin{tabular}{|c|c|c|}
\hline \multicolumn{3}{|c|}{ Altitudes (msnm) } \\
\hline \multirow[t]{5}{*}{1000} & $H T=0,669719^{\circ} \mathrm{D} \cdot 0,005471^{\circ} \mathrm{D}^{2}$ & Modelo 6 \\
\hline & $\lim \inf a=0,57426$ & $\lim$ sup $a=0,76517$ \\
\hline & $\lim \inf b=-0,00760$ & $\lim$ sup $b=-0,00334$ \\
\hline & $t a=14,3529 \cdots$ & $\mathrm{tb}=-5,2602 \cdots$ \\
\hline & Weigth $=1 / D^{2}$ & \\
\hline \multirow[t]{3}{*}{1100} & $H T=2,019294^{*} D^{0.5}$ & Modelo 8 \\
\hline & $\lim \inf =1,83356$ & $\lim$ sup $=2,20503$ \\
\hline & $\mathrm{ta}=22,0766 \cdots$ & Weigth $=1 / D^{2}$ \\
\hline \multirow[t]{5}{*}{1200} & $H T=1,483331 \cdot D^{0.5}+45,877077 / D$ & Modelo 10 \\
\hline & $\lim$ inf $a=1,30558$ & $\lim$ sup $a=1,66108$ \\
\hline & $\lim \inf b=29,1944$ & lim sup $b=62,5597$ \\
\hline & $\mathrm{ta}=17,0714 \cdots$ & tb $=5,6257 \cdots$ \\
\hline & Weigth $=1 / D^{2}$ & \\
\hline \multirow[t]{3}{*}{1300} & $H T=1,948446 \cdot D^{0.5}$ & Modelo 8 \\
\hline & $\lim$ inf $=1,76236$ & $\lim$ sup $=2,13454$ \\
\hline & $t_{a}=22,2019 \cdots$ & Weigth $=1 / D^{2}$ \\
\hline
\end{tabular}

$H T=$ Altura Total $(m)$

$\mathrm{D}$ = Diámetro a la altura del pecho $(\mathrm{cm})$

\section{$E S E=m$}

lim sup y $\lim$ inf $=$ Intervalos de confianza al $95 \%$ de los coeficientes calculados.

* altamente significativos al $95 \%$ de significancia 


\section{Cuadro $\mathrm{N}^{\circ} 12$}

\section{MODELOS FINALES SELECCIONADOS PARA AREA DE COPA PARA LA EXPOSICION ESTE}

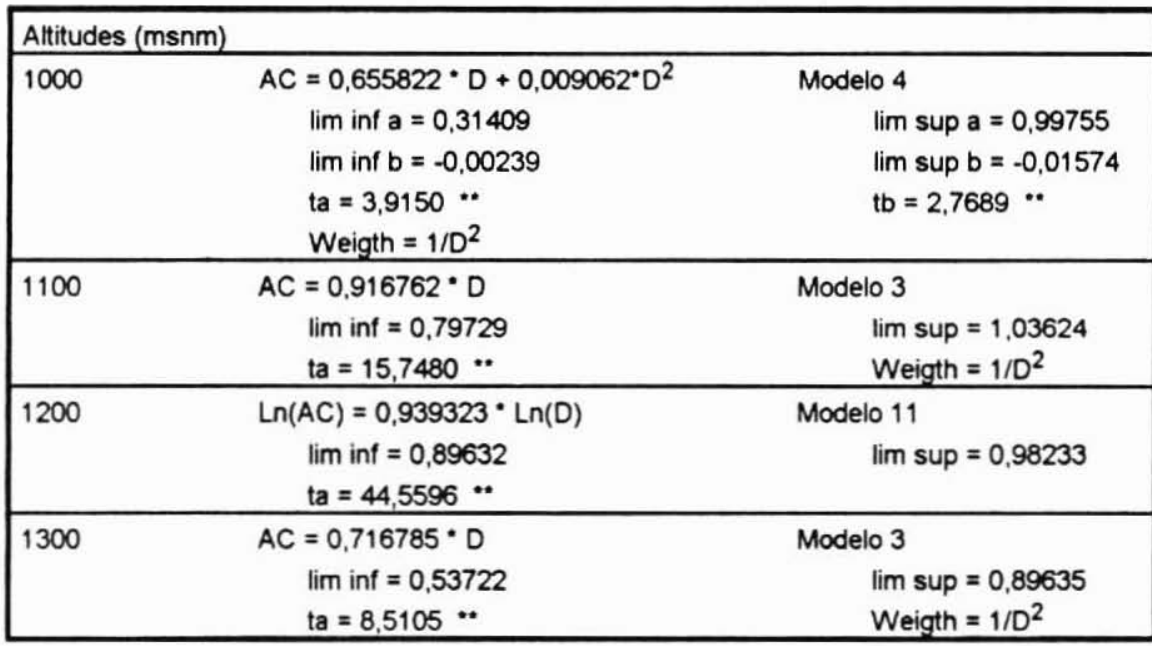

$A C=$ Area de Copa $\left(m^{2}\right)$

$D=$ Diámetro a la altura del pecho $(\mathrm{cm})$

$\mathrm{ESE}=\mathrm{m}^{2}$

lim sup y lim inf = Intervalos de confianza al $95 \%$ de los coeficientes calculados.

.* altamente significativos al $95 \%$ de significancia 


\section{Cuadro $\mathrm{N}^{\circ} 13$}

\section{MODELOS FINALES SELECCIONADOS PARA AREA DE COPA PARA LA EXPOSICION SUR}

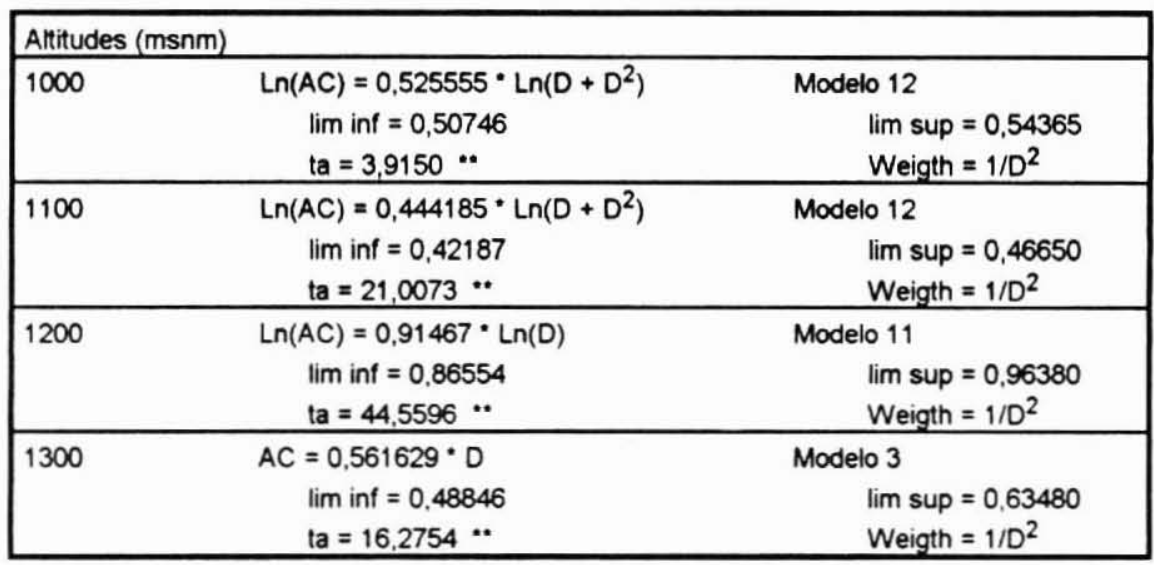

$A C=$ Area de Copa $\left(m^{2}\right)$

$D=$ Diámetro a la altura del pecho $(\mathrm{cm})$

$\mathrm{ESE}=\mathrm{m}^{2}$

$\lim$ sup y $\lim$ inf $=$ Intervalos de confianza al $95 \%$ de los coeficientes calculados.

* altamente significativos al $95 \%$ de significancia 
Cuadro $\mathrm{N}^{\circ} 14$

MODELOS FINALES SELECCIONADOS PARA AREA DE COPA PARA LA EXPOSICION NORTE

\begin{tabular}{|c|c|c|}
\hline \multicolumn{3}{|c|}{ Altitudes (msnm) } \\
\hline 1000 & $\begin{array}{c}A C=0,0,943747 \cdot D \\
\lim \text { inf }=0,81380 \\
\text { ta }=14,8357^{\cdots}\end{array}$ & $\begin{array}{l}\text { Modelo } 3 \\
\qquad \begin{aligned} \lim \text { sup } & =1,07369 \\
\text { Weigth } & =1 / D^{2}\end{aligned}\end{array}$ \\
\hline 1100 & $\begin{array}{c}A C=0,641764^{\circ} D \\
\lim \text { inf }=0,54943 \\
\text { ta }=14,1138^{\cdots}\end{array}$ & $\begin{array}{l}\text { Modelo } 3 \\
\qquad \begin{aligned} \lim \text { sup } & =0,73410 \\
\text { Weigth } & =1 / D^{2}\end{aligned}\end{array}$ \\
\hline 1200 & $\begin{array}{c}A C=0,553681 \cdot D \\
\lim \text { inf }=0,46850 \\
\text { ta }=13,2783 \cdots\end{array}$ & $\begin{array}{l}\text { Modelo } 3 \\
\qquad \begin{aligned} \lim \text { sup } & =0,63886 \\
\text { Weigth } & =1 / D^{2}\end{aligned}\end{array}$ \\
\hline 1300 & $\begin{array}{c}\operatorname{Ln}(A C)=0,826522^{\circ} \operatorname{Ln}(D) \\
\lim \text { inf }=0,78360 \\
\text { ta }=40,8337^{*}\end{array}$ & $\begin{array}{l}\text { Modelo } 11 \\
\qquad \begin{array}{l}\lim \text { sup }=0,86944 \\
\text { Weigth }=1 / D^{2}\end{array}\end{array}$ \\
\hline
\end{tabular}

$A C=$ Area de Copa $\left(\mathrm{m}^{2}\right)$

$D$ = Diámetro a la altura del pecho $(\mathrm{cm})$

$E S E=m^{2}$

lim sup y lim inf = Intervalos de confianza al 95\% de los coeficientes calculados.

* altamente significativos al $95 \%$ de significancia

En los gráficos de dispersión de los residuales se observó que los valores no siguen ningún patrón sistemático aparente. En los gráfico de valores predecidos - valores observados, en algunos modelos, se notó cierta dispersión no deseable de los valores observados con respecto a los ajustados.

Ningún residual normalizado superó el rango de $+/$ - tres desviaciones estándar, factor que permite inferir que todos los datos pertenecen a la misma población (Chauchard, 1991). 
Alcances de los modelos

Considerando los intervalos de confianza al $95 \%$ de los coeficientes calculados para los modelos de cada combinación de exposición y altitud, se determinó si existian diferencias significativas.

\section{Cuadro $\mathrm{N}^{\circ} 15$}

DETERMINACION DE LOS ALCANCES PARA LOS MODELOS DE ALTURA TOTAL PARA CADA COMBINACION DE EXPOSICION Y ALTITUD

\begin{tabular}{|c|c|c|c|c|c|c|c|c|c|c|c|c|}
\hline & $\mathrm{S10}$ & $\mathrm{S11}$ & $\mathrm{S} 12$ & $\mathrm{~S} 13$ & E10 & E11 & $\mathrm{E} 12$ & E13 & N10 & N11 & $\mathrm{N} 12$ & N13 \\
\hline $\mathrm{S} 10$ & $\cdot$ & $\mathrm{ns}$ & ns & . & ns & $\mathrm{ns}$ & $\mathrm{ns}$ & ns & ns & . & $\cdot$ & $\cdot$ \\
\hline S11 & ns & - & ns & - & - & ns & ns & - & ns & - & • & - \\
\hline $\mathrm{S} 12$ & ns & ns & - & ns & ns & ns & ns & ns & ns & ns & ns & ns \\
\hline $\mathrm{S} 13$ & - & - & ns & - & - & $\cdot$ & • & ns & • & $\cdot$ & ns & ns \\
\hline E10 & ns & $\cdot$ & $\mathrm{ns}$ & $\cdot$ & $\cdot$ & ns & $\cdot$ & $\overline{-}$ & ns & $\cdot$ & $\overline{-}$ & $\cdot$ \\
\hline E11 & ns & ns & ns & - & ns & - & ns & • & ns & - & - & - \\
\hline E12 & ns & ns & ns & - & - & ns & - & • & ns & - & • & • \\
\hline E13 & ns & $\cdot$ & ns & ns & - & - & - & ns & ns & ns & ns & ns \\
\hline N10 & ns & ns & ns & $\cdot$ & ns & ns & $\mathrm{ns}$ & ns & - & $\mathrm{ns}$ & ns & ns \\
\hline N11 & - & • & ns & • & - & • & • & ns & ns & - & ns & ns \\
\hline N12 & • & • & ns & ns & - & • & - & ns & ns & $\mathrm{ns}$ & - & ns \\
\hline $\mathrm{N} 13$ & - & • & ns & ns & - & - & - & ns & ns & ns & ns & - \\
\hline
\end{tabular}

Significativo al $95 \%$ de significancia

ns

No significativo

Dentro de una misma exposición se manifiesta un gradiente en la Altura Total que se evidencia a través de la significancia, tales como en las Este y Sur. En la exposición Sur los modelos a los 1100 y 1200 msnm son intermedios entre los modelos a los 1000 y $1300 \mathrm{msnm}$. En la exposición Este el modelo a los $1100 \mathrm{msnm}$ es intermedio entre los modelos a los 1000 y 1200 msnm. También existen diferencias significativas entre los modelos a 1200 y $1300 \mathrm{msnm}$. En la exposición Norte no se detectaron diferencias significativas entre las distintas altitudes.

Dentro de una misma altitud para diferentes exposiciones se detectaron 
diferencias significativas entre la exposición Sur con la Norte a los 1.100 msnm. No se detectaron diferencias significativas a los 1000 y $1300 \mathrm{msnm}$ entre las distintas exposiciones.

Entre distintas exposiciones y altitudes se presentaron pocas diferencias significativas al comparar las combinaciones correspondientes a Este y Sur, salvo las del gradiente altitudinal antes mencionado. Pero se detectó una mayor cantidad de diferencias significativas entre dichas combinaciones y las correspondientes a las de la exposición Norte.

\section{Cuadro $\mathrm{N}^{\circ} 16$}

DETERMINACION DE LOS ALCANCES PARA LOS MODELOS DE AREA DE COPA PARA CADA COMBINACION DE EXPOSICION Y ALTITUD

\begin{tabular}{|c|c|c|c|c|c|c|c|c|c|c|c|c|}
\hline & $\mathrm{S} 10$ & $\mathrm{~S} 11$ & $\mathrm{~S} 12$ & $\mathrm{~S} 13$ & E10 & E11 & E12 & E13 & N10 & N11 & N12 & N13 \\
\hline $\mathrm{S} 10$ & - & $\cdot$ & $\cdot$ & $\cdot$ & $\mathrm{ns}$ & $\cdot$ & $\bullet$ & $\cdot$ & $\cdot$ & $\cdot$ & $\cdot$ & $\bullet$ \\
\hline $\mathrm{S} 11$ & • & - & ns & ns & ns & ns & ns & ns & • & ns & • & • \\
\hline $\mathrm{S} 12$ & - & ns & - & ns & ns & ns & ns & ns & ns & ns & $\cdot$ & • \\
\hline $\mathrm{S} 13$ & - & ns & ns & $=$ & ns & - & - & ns & ns & ns & ns & ns \\
\hline E10 & ns & ns & ns & ns & - & ns & ns & ns & ns & ns & ns & ns \\
\hline E11 & - & ns & ns & • & ns & - & ns & ns & ns & • & $\cdot$ & • \\
\hline E12 & • & ns & ns & • & ns & ns & - & ns & • & • & ns & * \\
\hline E13 & - & ns & ns & ns & $\mathrm{ns}$ & ns & ns & - & ns & ns & ns & ns \\
\hline N10 & $\cdot$ & $\cdot$ & ns & $\mathrm{ns}$ & $\mathrm{ns}$ & ns & $\cdot$ & ns & - & $\cdot$ & $\bullet$ & $\cdot$ \\
\hline N11 & - & ns & ns & ns & ns & . & • & ns & * & - & ns & ns \\
\hline N12 & - & $\cdot$ & $\cdot$ & ns & ns & - & ns & ns & • & ns & - & ns \\
\hline N13 & - & - & - & ns & ns & - & $\dot{ }$ & ns & - & ns & ns & - \\
\hline
\end{tabular}

Significativo al $95 \%$ de significancia

ns : No significativo

Dentro de una misma exposición se manifiesta un gradiente en el tamaño del Area de Copa que se evidencia a través de la significancia, tales como en las Norte y Sur. En ambas exposiciones los modelos a los $1000 \mathrm{msnm}$ presentan diferencias significativas con los modelos de mayores altitudes. En la exposición Este no se presentan diferencias significativas para las distintas altitudes.

Dentro de una misma altitud para diferentes exposiciones se detectaron diferencias significativas entre la exposición Sur con la Norte a los 1000 y 1200 
msnm. La exposición Este con la Norte se diferencia a los $1100 \mathrm{msnm}$. No se detectaron diferencias significativas a los $1300 \mathrm{msnm}$ entre las distintas exposiciones.

Entre distintas exposiciones y altitudes se presentaron varias diferencias significativas al comparar las combinaciones correspondientes a Norte, Este y Sur, incluyendo las correspondientes al gradiente altitudinal antes mencionado. Sin embargo, se detectó una myor cantidad de diferencias significativas entre las correspondientess al Sur y al Norte.

\section{CONCLUSIONES}

Para el ajuste de ecuaciones de Altura Total, el modelo que mejor se adaptó es el potencial, de potencia $b=0.5$. Para el ajuste de ecuaciones de Area de Copa el modelo que mejor se adaptó es el lineal, con ordenada al origen igual a cero.

Con respecto a los estadísticos ensayados, los modelos presentaron distintos comportamientos en el ajuste. Un mejor ajuste y aptitud se observó en los modelos que fueron ponderados por la inversa del DAP elevado al cuadrado y, en consecuencia, el error estándar de la estimación disminuyó notablemente. Por otra parte, la presencia de coeficientes independientes en los modelos está asociada a los valores más bajos de los estadísticos y peor aptitud en el análisis del ANARE.

En cuanto a los modelos de Altura Total seleccionados, menos del $7 \%$ de la variación no es explicada por el DAP y sus transformaciones algebraicas. EI coeficiente de variación de los residuos varió entre 0,3 y $4 \%$. Para los modelos de Area de Copa, en tanto, menos del $18 \%$ de la variación no es explicada por el DAP y sus transformaciones algebraicas. El coeficiente de variación de los residuos osciló entre 0,01 y $3 \%$.

Dentro de una misma exposición se manifiesta un gradiente en la Altura Total y Area de Copa, lo que daría la pauta para el ajuste y uso de ecuaciones individualizadas por rango de altitud. La exposición Norte no presentó diferencias significativas en las diferentes altitudes para Altura Total. La exposición Este no presentó diferencias significativas en las diferentes altitudes 
para Area de Copa.

Dentro de una misma altitud se detectaron diferencias en los modelos de Altura Total y Area de Copa, lo que daria la pauta para el ajuste y uso de ecuaciones individualizadas por clases de exposición. La exposición Norte es la más disímil en relación a la Este y la Sur.

\section{RECONOCIMIENTOS}

Al Sr. Andres Nicolás Focke por su apoyo en la toma de datos de campo. Al Productor Forestal Sr. Nikita van Rennemkampf por el apoyo logístico y movilización en el lugar de la campaña.

\section{REFERENCIAS}

Alder, D., 1981. Estimación del Volumen Forestal y Predicción del Rendimiento con Referencia Especial a los Trópicos: Predicción del Rendimiento. Vol 22/2. FAO-Roma, Italia.

Bonnier, G. y Tedín, O., 1983. Bioestadistica, Los Métodos Estadisticos para la Elaboración de Experimentos Biológicos. Ed Acribia. España.

Cailliez, F., 1981. Estimación del Volumen Forestal y Predicción del Rendimiento con Referencia Especial a los Trópicos: Estimación del Volumen. Vol 22/1. FAO-Roma, Italia.

Cantatore de Frank, N., 1983. Manual de Estadistica Aplicada. Tomo II. Ed Hemisferio Sur. Argentina.

Carabelli, F. A., 1981. Estudio Bibliográfico sobre Lenga (Nothofagus pumilio (Poepp et Endl) Krassser, con Enfasis en Aspectos de Dinámica y Manejo del Bosque. Facultad de Ciencias Naturales. Universidad Nacional de la Patagonia. Centro de Investigaciones Forestales. Argentina. 
Clutter, J.; Fortson, J.; Pienaar, L.; Brister, G. y Bailey, R., 1983. Timber Management: a Quantitative Approach. Wiley \& Sons. EE.UU.

Chauchard, L., 1991. Familia de Funciones de Volumen de Lenga (Nothofagus pumilio (Poepp et EndI) Krasser). Actas de las VI Jornadas Técnicas : Inventarios Modelos de Producción y Crecimientos Forestales. Eldorado. Pag. 26-39. Argentina.

Donoso, C., 1985. Relaciones Vegetación-Altitud y Exposición en la Formación Forestal "Bosque Andino Abierto" en el Area de Bullileo. Departamento de Silvicultura. Universidad de Chile. Chile.

FriedI, R.; Costas, R.; Maiocco, D.; Grance, L. y Palavecino, J., 1991. Construcción de Tablas de Volúmenes Estándares para (Araucaria angustifolia (Bert) o Ktze. Actas de las VI Jornadas Técnicas: Inventarios-Modelos de Producción y Crecimientos Forestales. Eldorado. Pag. 57-68. Argentina.

Gaillard de Benítez, C.; Gallo de Ferrari, J. y Pece de Ríos, M., 1988. Determinación de Curvas de Altura en Función del Diámetro en Parcelas Experimentales de Alamos de la Estación Experimental San Carlos, Departamento Banda, Santiago del Estero. Actas del VI Congreso Forestal Argentino. Santiago del Estero. Pag. 22. Argentina.

Little, T. y Hills, J., 1985. Métodos Estadísticos para la Investigación en la Agricultura. Ed Trillas. México.

Moore, D., 1983. Flora of Tierra del Fuego. Publicado por Anthony Nelson (England) y Missouri Botanical Garden (USA).

Muttarelli, E. y Orfila, E., 1971. Plan de Investigaciones Silvo-Dasocráticas en las Etapas de Ordenación, Recuperación y Producción Económica de los Bosques AndinoPatagónicos. Informe Final. Convenio CAFPTA-Cátedra de Dasonomía (UBA). Inédito. Cátedra de Botánica, Fac de Ciencias Agrarias y Forestales UNLP).

Neter, J. y Wasserman, W., 1973. Fundamentos de Estadistica. Compañia Ed Continental. México.

Orfila, E., 1987. Consideraciones Dasométricas sobre (Nothofagus pumilio (Poepp et EndI) Krasser en la Zona del Lago General Vintter, Prov. del Chubut, Rep. Argentina. Jornadas sobre Nothofagus. Villa La Angostura, Neuquén. Argentina.

Puente, M.; Marín, G. y Cuevas, R., 1985. Modelos de Rodal para Bosques de (Nothofagus glauca (Phil) Krasser en Bullileo. Dpto. de Manejo de Recursos Forestales. Investigación y Desarrollo Forestal. Chile. 
Sokal, R y Rohlf, J., 1984. Introducción a la Bioestadistica. Ed Reverté. España.

Uriarte, A. y Grosse, H., 1991. Los Bosques de Lenga. Una Orientación para su Uso y Manejo (Recopilación Bibliográfica). Informe Técnico N 126. CORFO-INFOR. Chile.

Yamane, T., 1979. Estadistica. Ed Harla. México. 